\title{
Four methods of locating the egocenter: A comparison of their predictive validities and reliabilities
}

\author{
RAPHAEL BARBEITO and HIROSHI ONO \\ York University, 4700 Keele Street, Downsview, Ontario M3J IP3, Canada
}

\begin{abstract}
An attempt was made to identify the best method of locating the egocenter by comparing the predictive validity and reliability of the four methods introduced by Fry (1950), Funaishi (1926), Howard and Templeton (1966), and Roelofs (1959). To determine predictive validity, egocenters located by these methods were used to predict the responses of 14 subjects on three visual direction tasks; the correlation between the predicted and the actual responses on each task was computed. To determine reliability, the test-retest stability and the internal consistency were estimated for each method. All of the methods were reliable, but only the Howard and Templeton method predicted the results on all three of the visual direction tasks. The high reliability and predictive validity of the Howard and Templeton method is attributed to its high precision.
\end{abstract}

An important function of the human visual system is to provide information concerning the direction of objects in visual space, and in discussion of visual direction the concept of the egocenter ${ }^{1}$ is often invoked (e.g., Fry, 1950; Hering, 1879/1942; Ono, 1975). Inherent in this concept is the notion that the subjective directions of objects are judged from a single point, which is often assumed to be located midway on the interocular axis. This assumed location has been used when discussing the possible role of the egocenter in adaptive changes to sensory-motor conflict (Craske \& Crawshaw, 1974) and to account for adaptation in a specific sensory-motor task (Ono \& Angus, 1974). The location has been used also to describe eye movement patterns during changes in fixation (Hering, 1879/1942; Ono \& Nakamizo, 1977; Yarbus, 1957, 1967) and to explain the extent of apparent movement of a stimulus when the two eyes are alternately occluded (Ono \& Gonda, 1978).

Rather than assume a location for the egocenter, some investigators have measured the location. Individual differences in the measured location of the egocenter have been related to individual differences in patterns of eye movements (Pickwell, 1972, 1973) and to individual differences in the extents of apparent movement and perceived changes in visual direction during accommodative vergence (Ono, Wilkinson, Muter, \& Mitson, 1972). However, though these studies show the value of measuring the location of the egocenter, they used different methods of measurement.

This research was supported by Grant A0296 from the National Research Council of Canada to the second author. Reprint requests should be addressed to the second author. The authors wish to thank their many colleagues in visual perception at York University for their helpful comments on an earlier version of this paper.
There are four methods of locating the egocenter (Fry, 1950; Funaishi, 1926; Howard \& Templeton, 1966; Roelofs, 1959), but each yields a different mean location (Bailey, 1958, using Fry's method; Funaishi, 1926; Mather, 1969, using Howard and Templeton's method; Roelofs, 1959). Furthermore, there is no correlation among the locations yielded by the Funaishi, Roelofs, and Howard and Templeton methods (Mitson, 1972; Mitson, Ono, \& Barbeito, 1976), even though the Mitson et al. (1976) psychometric evaluation of these methods found that each was highly reliable in testretest stability and in internal consistency. Mitson et al. concluded that a decision as to which method locates the "true" egocenter must be based on predictive validity. If one method can be shown to yield egocenters that predict responses on various visual direction tasks better than do the other methods, it could be argued that the method is the most useful method of locating the egocenter.

The purpose of the present study is to determine the predictive validity of each of the four methods used to locate the egocenter. To determine predictive validity, the egocenter yielded by a modified version of each method was computed for each subject and used to predict his responses on a series of visual direction, or "criterion," tasks. The present study also reestimates the test-retest stability and internal consistency of the methods.

\section{METHOD}

\section{Methods of Locating the Egocenter}

A brief description of how the egocenter was located by each method is given below; more elaborate descriptions are given in Appendix A. For the Howard and Templeton method the subject aligned two stimuli to "himself." For the Funaishi method the subject pointed to the apparent direction of a 
fixated stimulus with respect to himself. For the Roelofs method the subject fixated the intersection of two lines that were coincident with the lines of sight and indicated a point on the illusory line produced by the two lines. For the Fry method the subject fixated one of two stimuli placed in the median plane and pointed to the apparent location of the diplopic images produded by the second stimulus. To estimate the location of the egocenter by the Howard and Templeton, Funaishi, and Roelofs methods, the procedure described for each was repeated for two directions of the stimuli. For Fry's method the procedure described yielded sufficient information. The stimuli were viewed binoculary for each method.

\section{Criterion Tasks}

Three criterion tasks were devised. Each was chosen with the assumption that the subject's responses would depend on the location of the egocenter.

Subjective median plane (SMP) task. This task requires judgment of the position of the straight-ahead. The subject is instructed to indicate verbally the point, on a scale, that appears directly in front of him. Judgments are made with the scale placed at two distances. The subject views the stimulus binocularly.

Three-point (3PT) task. This task requires judgments of the relative directions of three stimuli. The subject is instructed to judge the visual directions of two standard stimuli in the same frontal parallel plane and then adjust a third, closer stimulus so that its visual direction bisects the angle formed by the visual directions of the two standards. The task is repeated for two sets of directions of the standard stimuli. The subject views the stimuli binocularly.

Accommodative vergence (AV) task. This task requires judgment of the extent of apparent movement during accommodative vergence. Two stimuli are aligned to one eye, and the subject is instructed to change fixation from one stimulus to another and to indicate the extent of the apparent movement of the stimulus to which fixation is changed. This task is repeated for left- and right-eye alignments of the stimuli. Ono et al. (1972) have shown that the egocenters located by the Howard and Templeton method predict the difference in the extents of apparent movement between the left- and right-eye alignments of the stimuli. However, it is not known whether or not the egocenters located by the other methods can predict the outcome of this task. ${ }^{2}$

For all methods and tasks the subject sat at a specially constructed table with his head supported by an adjustable bite-board.

Each criterion task required additional apparatus and stimuli. For the SMP task the stimulus was a meter stick from which the subject read the point judged to be straight ahead. For each judgment the meter stick was placed in one of 16 predetermined positions in two frontal parallel planes. The planes were 40 and $90 \mathrm{~cm}$ from the corneal plane.

For the 3PT task three rods of $1-\mathrm{mm}$ diameter were used as the stimuli. Two sets of judgments were obtained. For each, the two standard stimuli were positioned 20 and $40 \mathrm{~cm}$ to one side of the median plane, in the frontal parallel plane $75 \mathrm{~cm}$ from the corneal plane. The movable stimulus was positioned in the frontal parallel plane, $25 \mathrm{~cm}$ from the corneal plane, and was mounted on a motorized drive unit so it could be driven to the subject's left or right.

For the AV task two point sources were used as stimuli. The near stimulus was placed in the median sagittal plane, $25 \mathrm{~cm}$ from the corneal plane. The far stimulus, which was to be aligned with the near stimulus and one eye, was positioned $50 \mathrm{~cm}$ from the corneal plane and was slightly above the height of the near stimulus. Once aligned, the far stimulus was occluded from the nonaligned eye to avoid diplopia. To indicate the extent of apparent movement of the far stimulus, the subject adjusted the distance between a movable and a stationary collar surrounding a rod until the distance between the two collars appeared to match the extent of the perceived movement. The rod was $30 \mathrm{~cm}$ long and $.5 \mathrm{~cm}$ in diamter. The collars were $1.5 \mathrm{~cm}$ in diameter and fit snugly around the rod.

\section{Procedure}

The experiment consisted of three sessions. In Sessions 1 and 3 the egocenter locations were measured by the four methods. The third session followed the first after 1 week and allowed for an estimation of the test-retest stability of each method. Session 2 was used to collect the criterion data and took place on the third day after the first session. Each session lasted approximately $1.25 \mathrm{~h}$, with subjects resting for $15 \mathrm{~min}$ after completing two methods or tasks.

There were eight trials for each egocenter-location method and each criterion task. For each egocenter-location method, a trial consisted of the minimum number of judgments necessary to estimate the location of the egocenter. The order of the judgments within a trial was counterbalanced for the eight trials. For each criterion task, a trial consisted of a single response, and the order in which the subject responded to the two stimulus arrangements for each task was counterbalanced for the eight trials. Subjects were given two practice trials before beginning each of the methods or tasks.

The order in which the subject did the four egocenter methods was randomly selected from the 24 possible orders. This order was repeated in the retest session (i.e., the third session). All subjects did the three criterion tasks in the same order.

The experiment was conducted under normal room illumination. The average luminance at the table top was $120 \mathrm{~cd} / \mathrm{m}^{2}$ Stimuli were $1.5 \mathrm{~cm}$ below eye level.

\section{Subjects}

Eight women and six men with normal visual acuity, either uncorrected or corrected with contact lenses, participated in the experiment. All were from the York University community and were paid for their participation.

\section{RESULTS}

For each of the four methods, the data from each trial provided an estimate of the egocenter's location. The estimated location was expressed as a point in a Cartesian plane lying horizontally through the pupils. The $x$-axis lay in the corneal plane of the subject and the $y$-axis in the median sagittal plane. The mean locations yielded by each of the methods, as well as analyses of the data, are presented in Appendix B.

\section{Reliability}

Two types of reliability were estimated for each method: test-retest stability and internal consistency. To estimate the test-retest stability, mean $X$ and mean $Y$ coordinate values were computed from the data of the eight trials of each session. The test-retest correlations for each of the mean egocenter coordinates from the two testing sessions are shown in Table 1. All are statistically significant $(p<.05)$. To estimate internal consistency, the trial-by-trial egocenter locations were considered analogous to 16 items on a test, and the consistency was estimated using Cronbach's alpha coefficient. This statistic is "the mean of all split-half coefficients resulting from different splittings of a test" 
Table 1

Reliabilities: Test-Retest Stability (Pearson r) and Internal Consistency (Cronbach $\alpha$ ) of Egocenter Dimensions Measured by Each Method

\begin{tabular}{llllll}
\hline & \multicolumn{2}{c}{$\begin{array}{c}\text { Test-Retest } \\
\text { Stability* }\end{array}$} & & \multicolumn{2}{c}{$\begin{array}{c}\text { Internal } \\
\text { Consistency }\end{array}$} \\
\cline { 2 - 3 } \cline { 5 - 6 } \multicolumn{1}{c}{ Method } & $\mathrm{X}$ & $\mathrm{Y}$ & & $\mathrm{X}$ & $\mathrm{Y}$ \\
\hline Fry & .52 & .85 & & .80 & .77 \\
Roelofs & .76 & .72 & & .90 & .86 \\
Funaishi & .67 & .63 & & .47 & .82 \\
Howard \& Templeton & .96 & .85 & .99 & .87 \\
\hline
\end{tabular}

*All correlations are statistically greater than $0(d f=12, p<.05)$.

(Cronbach, 1951). The coefficients, also shown in Table 1, are high, with the exception of one value for Funaishi's method. Thus, the results of the reliability analyses indicate that all four methods yield egocenter locations that are consistent both within and between testing sessions. These findings replicate those of Mitson et al. (1976), who evaluated the Funaishi, Roelofs, and Howard and Templeton methods, and establish that Fry's method is also a reliable method of locating the egocenter.

\section{Predictive Validity}

The predictive validity of each method of locating the egocenter was estimated by the correlation between the observed and predicted responses on each criterion task. The observed responses used in the analysis were the average of each subject's responses on the eight trials of each criterion task. The predicted responses were based on each subject's mean egocenter location, determined using the 16 trials of each method.

SMP task. Two sets of observed and predicted values were compared separately for each egocenter method. For each subject an estimate of the observed subjective median plane was determined at two frontal parallel planes. Also, two predicted values were determined from two subjective median planes, each being defined by the measured location of the egocenter and one of the points judged as being straight ahead. The point at which the subjective median plane, which included the near observed point, intersected the farther frontal parallel plane yielded a predicted far value that could then be compared with the observed far value. A comparable procedure was repeated to determine a predicted value at the nearer frontal parallel plane. The correlations for the observed and predicted values in each frontal parallel plane are shown in Table 2. All correlations are statistically significant, with the exception of one associated with Roelofs' method. The remaining correlations are similar and a decision as to which method yields the best estimates of the egocenter's location cannot be based on the data from this criterion.

3PT task. Each of the two sets of observed values were compared to the predicted values computed using the egocenters yielded by each method. For each subject, the predicted location of the movable stimulus (based on the egocenter yielded by each method) was determined by (1) computing the bisector of the angle formed by the egocenter and the two standard stimuli and (2) finding the point at which this bisector intersected the plane of movement of the movable stimulus. The correlations between the observed and predicted values are also shown in Table 2. Only Howard and Templeton's method yielded egocenters that successfully predicted this criterion, as indicated by the statistically significant correlations. The correlations for the other three methods were uniformly low and ranged from - .18 to .25 . Thus, for this criterion task, the Howard and Templeton method is clearly superior to the other methods of locating the egocenter.

AV task. The method of comparing observed and predicted values for this task was the same as that used by Ono et al. (1972). One set of observed values was compared to the predicted values computed using the egocenters yielded by each method. The observed value for each subject was the signed difference between his estimation of the extent of apparent movement for each of the two stimulus arrangements. The predicted value for each subject was the signed difference between two predicted extents. One extent is the distance between the actual position of one of the far stimuli and the point at which the far plane is intersected by the line that passes through the egocenter and the near stimulus; the other extent is the distance between the actual location of the other far stimulus and this intersection. The correlations between the observed and predicted values are also shown in Table 2. Only the Howard and Templeton method yielded egocenters that predicted the outcome of this criterion. The remaining correlations were not statistically greater than 0 and ranged from .18 to .38 . This criterion task is similar to Roelofs' method, yet the egocenters located by Roelofs' method could not predict the values observed. ${ }^{3}$

The results of the predictive validity analyses indicated that the Howard and Templeton method is clearly superior to the other methods in predicting two of the

Table 2

Predictive Validity: Correlation Between Predicted and Observed Values on Each Criterion

\begin{tabular}{|c|c|c|c|c|c|}
\hline \multirow[b]{3}{*}{ Method } & \multicolumn{2}{|c|}{ SMP } & \multirow{2}{*}{\multicolumn{2}{|c|}{ 3PT }} & \multirow[b]{3}{*}{ AV } \\
\hline & \multirow{2}{*}{$\begin{array}{l}\text { Near } \\
\text { Plane }\end{array}$} & \multirow{2}{*}{$\begin{array}{c}\text { Far } \\
\text { Plane }\end{array}$} & & & \\
\hline & & & Left & Right & \\
\hline Fry & $.47^{*}$ & $.55^{*}$ & -.18 & .25 & .38 \\
\hline Roelofs & .39 & $.61^{*}$ & .04 & -.01 & .33 \\
\hline Funaishi & $.53^{*}$ & $80^{*}$ & .04 & -.11 & .18 \\
\hline Howard \& Templeton & $.68^{*}$ & $.76^{*}$ & $.52 *$ & $.50^{*}$ & $.66^{*}$ \\
\hline
\end{tabular}

Note-In general, the variability of the values on the $X$ dimension of the egocenter measured by each method contributed most to the variability of the predicted values in the SMP and $A V$ criteria. The variability of the values on both the $X$ and $Y$ dimensions made approximately equal contributions to the variability in the $3 P T$ criterion. $\quad * d f=12, p<.05$. 
criteria. The fact that the Howard and Templeton method was the only method to yield egocenters that predicted the subjects' responses in all three criterion tasks strongly suggests that it is the best method of estimating the location of the egocenter.

\section{DISCUSSION}

The results of the present study are relevant to Fry's (1950) assertion that methods requiring judgments based on location of the self do not measure the egocenter, but rather measure an arbitrary point that the subject chooses to use as the self. Fry labels such methods "direct" and argues that "indirect" methods, which do not require the subject to select a reference point, should be used to locate the egocenter. In Fry's terminology, the Funaishi and Howard and Templeton methods are direct methods, and the Fry and Roelofs methods are indirect. The criterion tasks can also be thought of in this way. The SMP and 3PT are direct since they require use of the self as a reference point; the $\mathrm{AV}$ is indirect since it does not require reference to the self. Thus, in determining the best method of locating the egocenter, use of the SMP and 3PT tasks as criteria should favor the Funaishi and the Howard and Templeton methods; use of the AV task as a criterion should favor the Fry and the Roelofs methods. The difference implied by the distinction, however, is not evident in the predictive validity data. The Howard and Templeton method was the only one to predict all of the criteria. Thus, it appears that the point a subject uses as the self for the direct methods is related to the point from which visual directions are judged.

If the point subjects use as the self while doing the direct methods is equivalent to the location of the egocenter, an explanation for the relatively poor showing of the Funaishi method is necessary, because it too requires use of the self as a reference point. Furthermore, the fact that the Fry and Roelofs methods are indirect does not explain their inability to predict the criteria. The difference between the Howard and Templeton method and the other three methods is in the potential sources of error introduced by the modes of response required of the subject: Visual judgments are required for the Howard and Templeton method, and pointing responses based on visual judgments are required for the other three methods. The failure of three methods to predict the criteria may be the result of error associated with pointing, since the use of pointing responses is associated with high variability in the measured location of the egocenter. Estimates of a subject's egocenter yielded by the methods that use pointing are, without exception, more variable than estimates yielded by the Howard and Templeton method; the predictive validity may be reflecting differences in precision among the methods of locating the egocenter. According to this view, the inability of the Funaishi, Fry, and Roelofs methods to predict all of the criteria is the result of the relative imprecision of these methods rather than a lack of validity inherent in each method.

In addition to the variable error, each of the three methods may be affected by a constant error of pointing. The data from several studies conducted in our laboratory (Barbeito, 1976; Mitson, 1972; Mitson et al., 1976) have shown that the degree and direction of error in pointing varies among subjects. Such errors could have affected the data presented here in two ways. First, the variability due to individual differences in constant error could have masked the variability due to individual differences in the egocenter, thereby reducing the ability of these methods to predict the criteria. Second, the individual differences in constant error could have contributed to the reliability estimates of these methods; the relatively high reliability estimates obtained may be attributable to individual differences in constant error rather than to individual differences in the true location of the egocenter. Furthermore, the egocenters measured by these methods are differentially affected by constant error in pointing. For example, other things being equal, a given magnitude of constant error would cause the egocenter located by the Roelofs method to be more to the right than one located by the Funaishi method. Hence, the low correlations among the methods reported by Mitson et al. (1976) and observed in the present study could be explained.

Thus, while the results of this study clearly indicate that the Howard and Templeton method is the best method of locating the egocenter, they also suggest that its advantage over the other methods is one of greater precision (and possibly accuracy) rather than superior theoretical foundation.

\section{REFERENCES}

Balley, N. J. Locating the center of visual direction by a binocular diplopia method. American Journal of Optometry, $1958,35,484-495$.

BARBEITO, R. A. The importance of fixation in Bailey's and Roelofs' methods of locating the egocentre. Unpublished Master's thesis, York University, 1976.

Craske, B., \& Crawshaw, M. Adaptive changes of opposite sign in the oculomotor systems of the two eyes. Quarterly Journal of Experimental Psychology, 1974, 26, 106-113.

Cronbach, L. J. Coefficient alpha and the internal structure of tests. Psychometrika, 1951, 16, 297-334.

FolEY, J. M. Error in visually directed manual pointing. Perception \& Psychophysics, 1975, 17, 69-74.

Foley, J. M., \& HeLd, R. Visually directed pointing as a function of target distance. Perception \& Psychophysics, $1972,12,263-268$.

Fry, G. A. Visual perception of space. American Journal of Optometry, 1950, 27, 531-553.

FuNAISHI, S. Weiteres über das Zentrum der Sehrichtungen. Albrecht von Graefes Archiv für Ophthalmologie, 1926, 117, 296-303.

HERING, E. Spatial sense and movements of the eye (C. A. Radde, trans.). Baltimore: American Academy of Optometry, 1942. (Originally published, 1879.)

HowAR, I. P., \& TEMPletoN, W. B. Human spatial orientation. New York: Wiley, 1966. 
Mather, J. Visual direction and parameters of binocular space (doctoral dissertation, Bryn Mawr College, 1969). Dissertation Abstracts International, 1971, 31, 4367B. (University Microfilms No. 72-383)

Mitson, G. L. The measurement of the location of the egocentre. Unpublished Master's thesis, York University, 1972.

Mitson, G. L., ONo, H., \& Barbeito, R. Three methods of measuring the location of the egocentre: Their reliability, comparative locations and intercorrelations. Canadian Journal of Psychology, 1976, 30, 1-8.

Ono, H. Directions of objects seen from the cyclopean eye Saensu (Japanese edition of Scientific American), 1975, 5, 88-99. (The English translation appeared as Department of Psychology Reports, York University, Toronto, 1976, No. 29.)

ONo, H., \& ANgus, R. G. Adaptation to sensory-motor conflict produced by the visual direction of the hand specified from the cyclopean eye. Joumal of Experimental Psychology, 1974, 103, $1-9$.

Ono, H., \& Gonda, G. Apparent movement, eye movements and phoria when two eyes alternate in viewing a stimulus. Perception, 1978, 7, 75-83.

Ono, H., \& Naramizo, S. Saccadic eye movements during changes in fixation to stimuli at different distances. Vision Research, $1977,17,233-238$.

Ono, H., Wilkinson, A., Muter, P., \& Mitson, L. Apparent movement and change in perceived location of a stimulus produced by a change in accommodative vergence. Perception \& Psychophysics, 1972, 12, 187-192.

Pickwell, L. D. Hering's law of equal innvervation and the position of the binoculus. Vision Research, 1972, 12, 1499-1507.

Pickweld., L. D. Eye movements during the cover test. British Journal of Physiological Optics, 1973, 28, 23-25.

Roelofs, C. O. Considerations on the visual egocentre. Acta Psychologica, 1959, 16, 226-234.

YARBUS, A. L. Motion of the eye on interchanging fixation points at rest in space. Biophysics, 1957, 2, 679-683.

YARBus, A. L. Eye movements and vision. New York: Plenum, 1967.

\section{NOTES}

1. The concept of the egocenter has been variously referred to as the cyclopean eye, the center of visual direction, the binoculus, and the projection center.

2. A fourth criterion task was also used. The task required the subject to point to the apparent location of a stimulus viewed in a reduced cue situation. Based on the reports of Foley and Held (1972) and Foley (1975), it was expected that the direction of the stimulus would be currectly perceived even though its distance was misperceived. However, while some subjects demonstrated extents of misperceived distance comparable to those reported, the majority of subjects did not, and, as such, the observed extents of misperceived distance were insufficient to produce well-differentiated predicted locations of the stimulus based on the egocenter. This task will not be discussed further.

3 . At a reviewer's suggestion, the results from the two alignment conditions were analyzed separately; none of the correlations were significant. The lack of significant correlation is attributable to measurement error in the reports of apparent movement; the error may have inflated the variance of the reports. When the difference between the two alignment conditions is used, the effect of the measurement error in the criterion scores is reduced because the scores are based on twice as many observations.

\section{APPENDIX A}

\section{The Methods of Locating the Egocenter}

The Howard and Templeton method. This method requires the subject to fixate the front of a long rod in a horizontal plane at eye level. The subject is asked to rotate the rod until he judges it to be pointing directly at himself. The axis of the rod is then projected back toward the subject and is assumed to pass through the egocenter.

The Mitson et al. (1976) version of this method was used in the present study. For this version the rod is replaced by two stimuli at different distances from the subject. The stimuli may be thought of as replacing the front and back ends of the rod. The near stimulus could be moved left and right in the subject's frontal parallel plane. Thus, the stimulus configuration is conceptually identical to rotating the rod. Physiological diplopia is avoided by arranging that only one stimulus is visible at any given time. The subject controls which of the two stimuli is visible by means of a switch. Diplopia can not occur, provided the subject is correctly fixated on the single visible stimulus. The task for the subject is to switch fairly rapidly back and forth between the near and far stimuli and to move the near stimulus until the imaginary axis joining the two stimuli is judged to be pointing directly at himself. The procedure is repeated for different directions, and the intersection point of the projected axes is taken to be the egocenter located by the Howard and Templeton method.

For the present study, two stimulus directions were used. The stimuli were the metal-rod inserts of solenoids. The rods were $1 \mathrm{~cm}$ long and $1 \mathrm{~mm}$ in diameter. Two were positioned $75 \mathrm{~cm}$ from the corneal plane, $30 \mathrm{~cm}$ on either side of the median sagittal plane. A third was placed $25 \mathrm{~cm}$ from the corneal plane and was mounted on a motorized drive unit so that it could be driven to the subject's left or right. A switch was wired so that the subject could activate either the movable solenoid or one of the stationary solenoids. In this manner, only one stimulus was visible at any time. Switching time between the far and near solenoids was usually less that $1 \mathrm{sec}$.

The Funaishi method. This method requires the subject to fixate a point in the median plane and to judge the direction of nonfixated target stimuli placed to the right and left of the fixation point and in the same frontal parallel plane. The direction of the targets is indicated by having the subject point in their apparent direction. These pointing responses are constrained to two different distances from the subject and, while pointing, the hand is not visible to the subject. Lines are projected back through the response locations and are assumed to pass through the subject's egocenter.

The Mitson et al. (1976) version of this method was used in the present study. With the Funaishi method, only information about the apparent (not the objective) locations of the targets is ever used by the subject; where the subject is fixated is irrelevant. Consequently, for this version of Funaishi's method, the subject fixates the target rather than a point in the median plane. Each subject makes pointing responses to indicate the direction of the target with respect to himself. A line is projected backward through the two response locations toward the subject. This procedure is repeated for different directions of the target stimuli and the intersection of the projected lines is taken to be the egocenter located by the Funaishi method. Conceptually, there should be no difference between this procedure and that originally used by Funaishi (1926).

For the present study two stimulus directions were used. The stimuli were rods, $1 \mathrm{~mm}$ in diameter, inserted into the table top of the main apparatus. The fixation points were positioned $75 \mathrm{~cm}$ from the corneal plane, $30 \mathrm{~cm}$ on either side of the median sagittal plane. In the underside of the table top two grooves were cut and a calibrated rod with a handle was inserted into each groove. The rods moved freely to the subject's left or right, 25 and $50 \mathrm{~cm}$ from the corneal plane. The handles of these rods, which were used to specify the judgments of perceived direction, were $1.5 \mathrm{~cm}$ below the table top.

The Roelofs method. This method requires that the subject look with one eye at the front of a tube that is objectively aligned with the fovea of that eye. The other eye is occluded. According to Hering's (1879/1942) principles of visual direction, the tube will not appear to point to the eye, but rather it will 
appear to be along a radial line joining the subject's egocenter and the front of the tube. The apparent direction of the tube is defined as the projection from the front of the tube to a point on the subject's face toward which the tube appears to point. This projection is assumed to pass through the egocenter.

A potential problem arising with this method is that of phoria, which is the angular deviation of the occluded eye from the proper convergence angle. Ono, Wilkinson, Muter, and Mitson (1972) showed that under monocular viewing conditions the direction of points may be mislocalized as a result of phoria and that this problem could be minimized by moving the occluder so that the eye is only partially occluded (i.e., the fixated point is viewed binocularly).

For this reason, Mitson et al. (1976) modified Roelofs' method. For this version of Roelofs' method, the tube is replaced by two point stimuli located at different distances. The two stimuli are aligned with the fovea of the nonoccluded eye. The other eye is partly occluded in such a way that it can see the near but not the far stimulus. The subject fixates the near stimulus; it is assumed that this stimulus is correctly localized. According to Hering's principles of visual direction, the apparent position of the far point stimulus is on a radial line joining the subject's egocenter and the point of fixation (in this case, the near stimulus). The subject indicates the apparent location of the far stimulus. Pointing is constrained to a frontal parallel plane and, while pointing, the hand is not visible to the subject. A line is projected from this apparent location through the actual location of the near stimulus toward the subject. This line is assumed to pass through the egocenter. This procedure is conceptually identical to Roelofs' method. The procedure is repeated for different directions of the stimuli, and the intersection point is taken to be the egocenter located by Roelofs' method.

There is an additional problem with Roelofs' method that the Mitson et al. version does not circumvent. The results of Barbeito (1976) showed that imprecisions in fixation exist and that these imprecisions result in invalid estimates of the egocenter's location. Thus, additional modification of this method was necessary. The modification was designed to remove the imprecisions in fixation.

For further modification of Roelofs' method, the subject fixates a point at the intersection of two lines coincident with his lines of sight. In this viewing situation a third, illusory line is perceived. According to one of Hering's principles of visual direction, this line points to the egocenter. The subject is to indicate a point on the illusory line. While indicating the location of the point, the hand is not visible to the subject. This point and the fixation point estimate a line passing through the egocenter. This procedure is repeated for different directions and the intersection of the two lines is taken to be the egocenter located by Roelofs' method. The advantage of the revised method is that the subject has information about the precision of his fixation via the percept of the line stimuli. If incorrectly fixated, the subject perceives four lines instead of the three perceived when correctly fixated.

For the present study two stimulus directions were used. The fixation stimuli were the same as those used for the Funaishi method. Also, from each fixation point, a line extended in the direction of each of the foveas to the edge of the table top, $8.9 \mathrm{~cm}$ from the corneal plane. The subject indicated a point on the illusory line using the rod handle under the table top, $25 \mathrm{~cm}$ from the corneal plane.

The Fry method. This method requires the subject to fixate the farther of two stimuli positioned in his median sagittal plane and to indicate the apparent location of each diplopic image produced by the nonfixated stimulus. While indicating the locations of the diplopic images, the hand used is not visible to the subject. To locate the egocenter by this method, Fry (1950) relied on two of Hering's principles of visual direction that specify the relationship among the location of the egocenter, the perceived direction of the fixation stimulus, and the perceived direction of each diplopic image.
Table 3

Means and Standard Deviations (in Centimeters) of the $X$ and $Y$ Coordinate Values Yielded by Each of the Methods

\begin{tabular}{lrrrrr}
\hline & \multicolumn{2}{c}{$X$} & & \multicolumn{2}{c}{$Y$} \\
\cline { 2 - 3 } \cline { 5 - 6 } \multicolumn{1}{c}{ Method } & Mean & SD & & Mean & SD \\
\hline Fry & .28 & 1.44 & & -15.07 & 7.95 \\
Roelofs & .27 & 1.18 & & .99 & 2.36 \\
Funaishi & -.29 & .90 & -2.69 & 4.10 \\
Howard \& Templeton & .28 & .97 & -1.16 & 1.16 \\
\hline
\end{tabular}

Note-Positive $X$ and $Y$ values indicate a location in front of the corneal plane and to the right of the median sagittal plane.

As in the case of Roelofs' method, Fry's method is susceptible to errors in fixation. Barbeito (1976) has shown that the inaccuracies and imprecisions in fixation result in invalid estimates of the egocenter's location. So Fry's method was modified in the following manner. To improve fixation, a line is positioned in the subject's median sagittal plane which extends from the fixation point toward the subject. When the subject fixates the farther stimulus, the line is perceived as two lines forming a $V$ shape, with the vertex of the $V$ at the fixation point. This "V" percept occurs when the subject is correctly fixated. Thus, with this addition to the stimulus display, the subject has information concerning the state of his fixation.

The fixation stimulus was a $1-\mathrm{mm}$-diam rod inserted in the table top, $75 \mathrm{~cm}$ from the corneal plane. The second stimulus was a $1.0 \times .6 \mathrm{~cm}$ diam cylinder placed $25 \mathrm{~cm}$ from the corneal plane. The single line in the median sagittal plane extended from the fixation point to the edge of the table top, $8.9 \mathrm{~cm}$ from the corneal plane. The apparent location of each diplopic image was indicated by inserting a push pin into a specially constructed board mounted on the underside of the table. The distance between the pointing surface of the board and the table top was $3 \mathrm{~cm}$.

\section{APPENDIX B}

\section{Analysis of Data}

For each subject the mean of the 16 trial-by-trial egocenters was taken to be the location of the egocenter estimated by each method. The locations were expressed in $X$ and $Y$ coordinate values, and separate analyses of variance were performed to determine whether these methods yielded different egocenters. The $F$ values were $F(3,39)=1.28, p>.05$, and $F(3,39)=31.32$, $\mathrm{p}<.01$, for the $\mathrm{X}$ and $\mathrm{Y}$ coordinates, respectively. Orthogonal comparisons indicated that the significant between-group difference was attributable to the fact that the egocenters located by Fry's method differed from the other three. The overall mean and standard deviation of the locations yielded by each method is shown in Table 3.

The overall mean location yielded by a given method in one experiment may not be meaningfully compared to that yielded in another experiment. The extent to which they are comparable depends on the extent to which measurement error associated with the method affected the mean in each study. The difficulty of comparison across experiments is exemplified by the location determined by Roelofs' method. Mitson et al. (1976) reported that this method located the egocenter $17.23 \mathrm{~cm}$ behind the corneal plane; the present study reported it $.99 \mathrm{~cm}$ in front of the plane. In the present study attempts to control fixation were made; however, such attempts were not made by Mitson et al. Since the egocenters located by this method are affected by errors in fixation (Barbeito, 1976), the discrepancy in the mean location reported by these two studies is likely the result of failure to control fixation error in the Mitson et al. study.

Received for publication August 24, 1978; revision accepted December 28,1978 .) 